\title{
Prevalencia y factores asociados a publicar artículos científicos durante la residencia médica en Perú
}

Percy Herrera-Añazco, Pedro Ortiz-Saavedra, Álvaro Taype-Rondán, Wendy Nieto-Gutiérrez, Carlos Alva-Díaz, David Jumpa-Armas, Seimer Escobedo-Palza, Raúl Timaná-Ruiz, Consejo Nacional de Residentado Médico (CONAREME)
Objetivo. Determinar la prevalencia y los factores asociados a publicar artículos científicos en médicos residentes de Perú. Sujetos y métodos. Estudio transversal analítico usando los datos obtenidos por la Encuesta Nacional para Médicos Residentes 2016 (ENMERE-2016), realizada virtualmente durante junio de 2016. Población: los médicos que realizan su residencia en alguna universidad de Perú. Muestra: los residentes que voluntariamente participaron en la ENMERE-2016. Desenlace de interés: autorreporte de haber publicado al menos un artículo científico. Se recogieron variables sociodemográficas, de la universidad, de la sede, de la residencia, de la percepción del residente y de síntomas depresivos. Para evaluar los factores asociados a haber publicado algún artículo científico se calcularon razones de prevalencia crudas y ajustadas con sus intervalos de confianza al 95\%.

Resultados. Se analizaron datos de 1.062 residentes $(42,2 \%$ de sexo femenino), de los cuales 118 (11,1\%) publicaron algún artículo científico durante la residencia. En el análisis ajustado, cursar los años superiores de la residencia, haber realizado alguna rotación externa fuera del país y tener una puntuación más alta en la opinión global del proceso de formación fueron variables directamente asociadas con haber publicado algún artículo científico, en tanto que el sexo femenino, tener más de 35 años y presentar síntomas depresivos fueron variables inversamente asociadas a dicho desenlace.

Conclusión. Aproximadamente uno de cada 10 residentes publicó algún artículo científico durante la residencia. Esto fue menos prevalente en las mujeres, en los residentes de mayor edad, en quienes cursaban años inferiores de la residencia, en quienes no realizaron ninguna rotación fuera del país, en quienes tuvieron una peor opinión del proceso de formación y en quienes presentaban síntomas depresivos.

Palabras clave. Educación médica. Internado y residencia. Investigación. Perú.

\section{Prevalence and associated factors to publish scientific articles during the medical residency in Peru}

Aim. To determine the prevalence and the factors associated with the publication of scientific articles in medical residents of Peru.

Subjects and methods. Cross-sectional analytical study using the data obtained by the National Survey for Resident Physicians 2016 (ENMERE-2016), carried out virtually during June 2016. Population: physicians who live in a university in Peru. Sample: residents who voluntarily participated in ENMERE-2016. Outcome of interest: have published at least one scientific article. Sociodemographic variables were collected, from the university, from the headquarters, from the residence, from the resident's perception, and from depressive symptoms. To evaluate the factors associated with having published a scientific article, crude and adjusted prevalence ratios were calculated with their $95 \%$ confidence intervals.

Results. Data from 1062 residents (42.2\% female) were analyzed, of which 118 (11.1\%) published some scientific paper during the resident. In the adjusted analysis, to study the superior years of residency, to have performed some external rotation outside the country and to have a higher score in the overall opinion of the training process were variables directly associated with having published some scientific article. While the female sex, being over 35 years old and presenting with depressive symptoms were variables inversely associated with this outcome.

Conclusion. Approximately one in ten residents published a scientific article during the residency. This was less prevalent in women, in older residents, in those who were in lower years of residence, in those who did not perform any rotation outside the country, in those who had a worse opinion of the training process, and in those with depressive symptoms.
Universidad San Ignacio de Loyola (P. Herrera-Añazco). Facultad de Medicina; Universidad Peruana Cayetano Heredia (P. Ortiz-Saavedra). CRÓNICAS, Centro de Excelencia en Enfermedades Crónicas; Universidad Peruana Cayetano Heredia (A. TaypeRondán). Facultad de Medicina Humana; Universidad de San Martín de Porres (W. Nieto-Gutiérrez). Red de Eficacia Clínica y Sanitaria, REDECS (C. Alva-Díaz, D. JumpaArmas). International Society For Pharmacoeconomics and Outcomes Research, ISPOR; capítulo Perú (C. Alva-Díaz, S. Escobedo-Palza). IBT Health; Hospital Guillermo Kaelin de la Fuente (D. Jumpa-Armas). Sociedad Peruana de Administración en Salud (S. Escobedo-Palza). Instituto de Evaluación de Tecnologías en Salud en Investigación; EsSalud (R. Timaná-Ruiz). Lima, Perú.

Correspondencia:

Dr. Percy Herrera Añazco.

Olavegoya, 1879. Dpto. 701 Jesús María, Lima, Perú.

E-mail:

silamud@gmail.com

Recibido:

02.05.17.

Aceptado:

08.05.17.

Conflicto de intereses: No declarado.

Competing interests: None declared.

(c) 2018 FEM

Key words. Education medical. Internship and residency. Peru. Research. 


\section{Introducción}

La investigación es un componente importante durante la formación del médico especialista. Su práctica permite lograr un mejor manejo clínico de los pacientes, desarrollar habilidades de apreciación crítica de artículos científicos, fomentar el pensamiento crítico y del aprendizaje permanente, y tener una mayor satisfacción con su formación académica [1-6]. Para ello se han diseñado estrategias para impulsar la investigación durante la residencia, muchas de las cuales han tenido resultados exitosos en términos de producción científica y del prestigio de los programas académicos donde se han llevado a cabo $[7,8]$.

Toda investigación debería culminar con una publicación en revistas científicas indexadas con la finalidad de asegurar su calidad y difundir los resultados obtenidos $[9,10]$. Sin embargo, el porcentaje de residentes que han publicado algún artículo varía de acuerdo con el país y la sede de la residencia estudiada, con diferencias tan amplias como el 5\% y el $21 \%$ entre residentes norteamericanos que no han realizado y han realizado una rotación en investigación, respectivamente $[11,12]$, hasta proporciones tan altas como el 59,2\% en el mismo país [6]. Por otro lado, la proporción de residentes con alguna publicación fue del 28,2\% en Galicia [13] y del 5\% entre los residentes de pediatría en Argentina [14]. En Perú, una evaluación entre residentes de una universidad privada mostró que son escasas las publicaciones científicas realizadas por residentes y éstas generalmente se encuentran en revistas de bajo impacto [15]; incluso una muestra de residentes y egresados de nefrología encontró que ninguno de ellos había publicado artículos científicos durante la residencia [16].

En Perú, los programas de residencia que incluyen asignaturas de investigación no tienen el objetivo de fomentar el desarrollo y la publicación de artículos científicos [16]. Asimismo, un requisito para obtener el título de especialista era la sustentación de una tesis de investigación, lo que se eliminó en 2015 [5]. Esto desató debates sobre los pros y contras de tal medida $[5,17]$ en el contexto peruano, caracterizado por su baja producción científica $[18,19]$.

Debido a la importancia de la publicación científica durante la residencia, y dado que los estudios sobre publicación científica en residentes de Perú se han realizado en subgrupos de una especialidad o de una universidad, el objetivo del presente estudio fue determinar la prevalencia y los factores asociados a publicar artículos científicos entre médicos residentes de Perú.

\section{Sujetos y métodos}

\section{Diseño de estudio}

Estudio transversal analítico que usó los datos obtenidos por la Encuesta Nacional para Médicos Residentes 2016 (ENMERE-2016), la cual fue realizada en junio de 2016 a médicos residentes de Perú.

\section{Población}

La población de estudio estuvo conformada por los médicos que realizan su residencia en alguna universidad de Perú. La muestra la constituyeron los que voluntariamente participaron en la ENMERE2016. Se excluyó a quienes no brindaron información sobre el desenlace de interés.

\section{Procedimientos}

En Perú, los médicos que deseen realizar la residencia deben postular a las plazas ofrecidas por alguna de las universidades acreditadas por el Comité $\mathrm{Na}$ cional del Residentado Médico del Perú (CONAREME). Para 2016, fueron siete las universidades acreditadas en Lima y 16 en otras ciudades.

Los médicos que ingresan a la residencia eligen una sede, que suele ser el hospital donde realizarán la mayor parte de su residencia. Estas sedes las administra alguno de los siguientes organismos: el Ministerio Nacional de Salud (MINSA) y los gobiernos regionales, el Ministerio de Trabajo (Seguro Social de Salud-EsSalud), el Ministerio del Interior (sanidades) o entidades privadas. El MINSA y los gobiernos regionales administran la mayor parte de las sedes de residencia.

El CONAREME diseñó la ENMERE-2016, que fue puesta a disposición de los médicos residentes de Perú de manera virtual desde la página web de CONAREME durante junio de 2016. Se invitó a todos los residentes de Perú a participar de esta encuesta mediante los correos electrónicos personales registrados en la base de datos del CONAREME, así como por publicaciones en Facebook y por periódicos de circulación local.

En la plataforma virtual se solicitó el número del documento nacional de identidad del residente. Posteriormente se mostró un texto que recalcaba la voluntariedad de la encuesta y la confidencialidad de los datos. De aceptar participar, se mostraron las preguntas de la encuesta. Además, se puso a disposición de los residentes una dirección de correo electrónico para que los residentes pudieran enviar las dudas que tuvieran sobre el desarrollo de la en- 
cuesta. Los resultados se guardaron en la base de datos del servidor del CONAREME.

La encuesta estuvo a disponibilidad de los residentes para su llenado durante el mes de junio de 2016 debido a que la mayor parte de residentes en Perú termina de trabajar o pasa al siguiente año el primer día de julio, y se buscó recolectar información correspondiente al último año transcurrido de la residencia.

Se preguntó a los residentes sobre el número de artículos publicados en revistas científicas durante su residencia. Se incluyeron todos los artículos publicados en revistas científicas, como artículos originales, casos clínicos y cartas al editor, entre otros. El desenlace de interés es haber publicado al menos un artículo científico durante la residencia.

Se recogieron las siguientes variables sociodemográficas: sexo (masculino o femenino), edad (en terciles), soportar dependientes económicos (sí o no) y tener especialidad previa (sí o no). Se recolectaron variables sobre la universidad y sede: tipo de universidad (universidad pública de Lima, universidad privada de Lima, universidad pública de otras ciudades o universidad privada de otras ciudades) e institución a la que pertenece su sede de residencia (MINSA y gobiernos regionales, EsSalud u otros). Se recolectaron variables sobre la residencia: modalidad (libre, que es adjudicada por concurso público, o por destaque/cautiva, que es adjudicada entre médicos que ya tienen un contrato), tipo de especialidad (clínica, quirúrgica u otras), año en curso (primero, segundo o tercero a más), horas que trabaja al día sin contar guardias $(\leq 8,>8$ a $<13$ $o \geq 13$ ) y haber realizado alguna rotación fuera de Perú (sí o no). Se evaluó la percepción del residente sobre su opinión global del proceso de formación, para lo cual se le pidió seleccionar un número del 0 al 9, siendo 0 una formación pésima y 9 una formación excelente. Además, se evaluó la presencia de síntomas depresivos mediante el Patient Health Questionnaire-2, con un punto de corte $\geq 2$ [20].

\section{Análisis estadísticos}

Para presentar descriptivamente los datos se usaron frecuencias absolutas y relativas. Para evaluar los factores asociados a haber publicado algún artículo científico se calcularon razones de prevalencia crudas y ajustadas con sus intervalos de confianza al 95\% mediante regresiones de Poisson con varianza robusta. El modelo ajustado incluyó todas las variables que resultaron significativamente asociadas al desenlace de interés en el modelo crudo. Todos los análisis se realizaron con el programa STATA v. 14.

\section{Aspectos éticos}

El presente estudio es un análisis secundario que fue aprobado por el comité de ética del Hospital Nacional Docente Madre Niño San Bartolomé (RCEI$40)$, de manera que no se tuvo contacto con los sujetos de estudio. Se cuidó la confidencialidad de los datos, que solo fueron administrados por los evaluadores.

\section{Resultados}

Durante junio de 2016, CONAREME registró un total de 7.393 médicos que se encontraban realizando su residencia en Perú, de los cuales 1.269 ingresaron a la plataforma virtual para completar la ENMERE-2016. De éstos, se eliminó a 207 participantes por no haber brindado datos sobre el desenlace de interés, y quedaron 1.062 participantes $(14,4 \%)$ para el análisis final.

Los residentes cuyos datos fueron analizados difirieron del total de residentes en cuanto a la universidad, la sede, la especialidad y el año de residencia (Tabla I).

De los 1.062 residentes evaluados, el $42,2 \%$ era del sexo femenino y el $28,2 \%$ tenía de 35 a 55 años. El 64\% estaba realizando su residencia en Lima. El $65,9 \%$ eran residentes en hospitales del MINSA y gobiernos regionales, el $81,8 \%$ había ingresado por modalidad libre, el 49,4\% eran residentes de especialidades clínicas, y el 40,9\% estaba cursando el primer año de residencia (Tabla II).

En cuanto a la prevalencia de haber publicado algún artículo científico durante la residencia, 118 $(11,1 \%)$ publicaron algún artículo, de los cuales 80 $(7,5 \%)$ publicaron un artículo, 24 (2,3\%) publicaron dos artículos y 14 (1,3\%) publicaron tres o más artículos durante su residencia.

Tras el análisis ajustado, cursar los años superiores de la residencia, haber realizado alguna rotación externa fuera del país y tener una puntuación más alta en la opinión global del proceso de formación fueron variables directamente asociadas con haber publicado algún artículo durante la residencia; en tanto que el sexo femenino, tener más de 35 años y presentar síntomas depresivos fueron variables inversamente asociadas con este desenlace (Tabla III).

El 40,1\% de la población ha realizado algún proyecto de investigación durante la residencia, el 16\% ha realizado algún trabajo de investigación durante la residencia, el $51,8 \%$ se siente capacitado para realizar un trabajo de investigación y el 44,3\% se sien- 
Figura. Prevalencia de autorreporte de actividades relacionadas con la investigación durante la residencia.

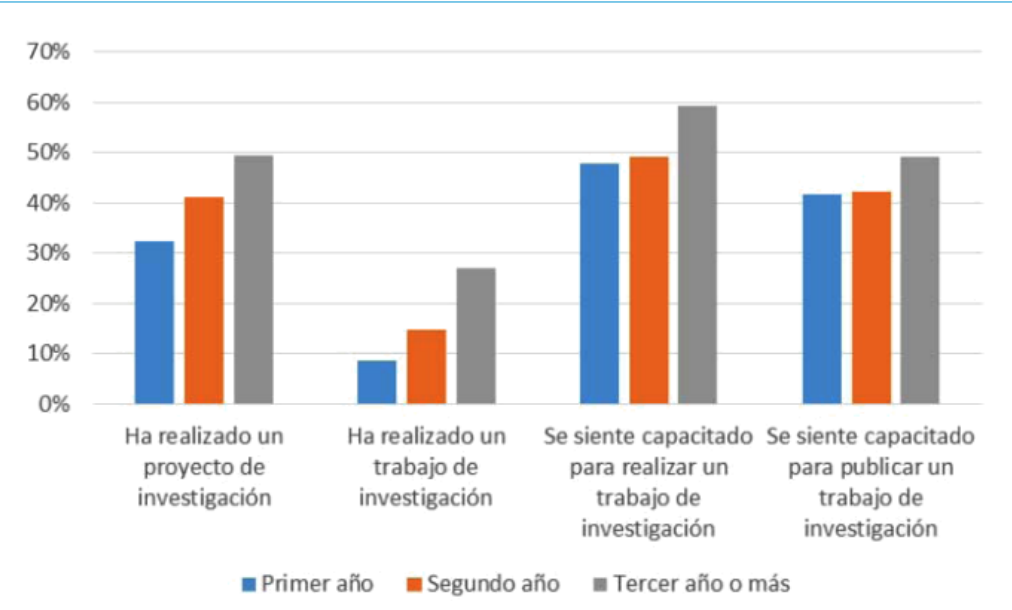

Tabla I. Diferencias entre el total de residentes registrados en la base del CONAREME y los residentes incluidos en el presente estudio.

\begin{tabular}{|c|c|c|c|c|c|}
\hline & & $\begin{array}{l}\text { Total de } \\
\text { residentes } \\
(n=7.393)\end{array}$ & $\begin{array}{l}\text { Residentes } \\
\text { incluidos } \\
(n=1.062)\end{array}$ & $\begin{array}{c}\text { Porcentaje } \\
\text { de } \\
\text { incluidos }\end{array}$ & $p\left(\chi^{2}\right)$ \\
\hline \multirow{4}{*}{ Universidad } & Lima, pública & 2.628 & 307 & $11,7 \%$ & \multirow{4}{*}{$<0,001$} \\
\hline & Lima, privada & 2.551 & 370 & $14,5 \%$ & \\
\hline & Provincia, pública & 1.546 & 237 & $15,3 \%$ & \\
\hline & Provincia, privada & 668 & 144 & $21,6 \%$ & \\
\hline \multirow{3}{*}{ Sede } & MINSA y gobiernos regionales & 4.573 & 697 & $15,2 \%$ & \multirow{3}{*}{0,041} \\
\hline & EsSalud (seguro social) & 2.208 & 284 & $12,9 \%$ & \\
\hline & Otros & 612 & 77 & $12,6 \%$ & \\
\hline \multirow{3}{*}{ Especialidad } & Médica & 3.704 & 523 & $14,1 \%$ & \multirow{3}{*}{0,018} \\
\hline & Quirúrgica & 2.786 & 374 & $13,4 \%$ & \\
\hline & Otros & 903 & 161 & $17,8 \%$ & \\
\hline \multirow{3}{*}{$\begin{array}{l}\text { Año de } \\
\text { residencia }\end{array}$} & Primero & 2.713 & 433 & $16,0 \%$ & \multirow{3}{*}{$<0,001$} \\
\hline & Segundo & 2.642 & 287 & $10,9 \%$ & \\
\hline & Tercero o más & 2.038 & 338 & $16,6 \%$ & \\
\hline
\end{tabular}

MINSA: Ministerio de Salud.

\section{Discusión}

\section{Resultados principales}

El 11,1\% de la población evaluada manifestó haber publicado algún artículo durante la residencia. Esto fue mayor en residentes de años superiores, en quienes realizaron alguna rotación externa internacional y en quienes otorgaron mayor puntuación a su proceso de formación. Por el contrario, ser de sexo femenino, tener más de 35 años y presentar síntomas depresivos fueron factores asociados a menor prevalencia de haber publicado algún artículo científico durante la residencia.

\section{Investigación científica en la residencia}

Cerca de uno de cada 10 de los residentes evaluados ha publicado algún artículo científico, y en la mayoría de los casos sólo fue un artículo. Estos resultados se encuentran por debajo de lo encontrado en otros escenarios. En Galicia se encontró que el $28,2 \%$ de los residentes de medicina interna publicó algún artículo científico [13], y en Arabia Saudí la proporción de residentes que ha publicado era del $17,2 \%$ [21]. Sin embargo, es más alta que la encontrada en residentes de pediatría chilenos, donde la proporción de publicación de artículos científicos sólo fue del 5\% [14]. En Perú, a pesar de que el $32,5 \%$ de los residentes y egresados de nefrología a nivel nacional había realizado algún trabajo de investigación, ninguno lo publicó [16].

No obstante la poca frecuencia de artículos científicos publicados entre los residentes evaluados, el $50 \%$ y el $25 \%$ de los residentes de tercer año han realizado algún proyecto o algún trabajo de investigación, respectivamente. Esta participación en actividades de investigación está por encima de algunas publicaciones internacionales; así, el 18\% de los residentes de pediatría del Children's Hospital Boston [10], el 19,9\% de residentes de medicina física y rehabilitación en Estados Unidos [9] y el 30,4\% en Arabia Saudí [21] estuvieron envueltos en alguna investigación científica. Estas discrepancias pueden deberse a las distintas definiciones usadas en los estudios descritos o al hecho de que hasta el año 2015 una de las exigencias de las universidades peruanas para otorgar el título de especialista era presentar un trabajo de investigación al finalizar la residencia, lo cual animaba a los residentes a realizar una investigación. Sin embargo, es posible que, ahora que dicho requisito ha desaparecido, el número de residentes que realicen proyectos y trabajos de investigación disminuya. 
Las tutorías en investigación en Perú son insuficientes o se destinan a la realización de la tesis y no a la publicación de artículos científicos [16]. Esta falta de tutoría especializada al parecer es un problema que la educación médica peruana sufre desde pregrado, al constatarse la pobre producción científica de los profesores de cursos de investigación en las facultades de medicina peruana [22]. En un estudio realizado en un centro médico del medio oeste en Estados Unidos, la participación de un editor médico asesor aumentó las publicaciones de residentes de seis en 2009 a 53 en 2012 [23], y en Perú, contar con un tutor con publicaciones científicas aumenta las posibilidades de que un residente culmine su tesis de grado [24]. En ese sentido, un entorno académico estimulante, que incluya tutores con publicaciones científicas verificables en las sedes de residencia, podría ser un objetivo que se debería buscar si es que se desea aumentar la producción científica entre nuestros residentes [25].

\section{Factores asociados a la producción}

científica entre residentes

Entre los factores asociados a haber publicado algún artículo científico, el año de residencia fue uno de los más destacados, lo cual se explica debido a que se preguntó por los artículos publicados durante la totalidad de la residencia; es decir, el tiempo de estudio en los residentes de primer año fue de un año, mientras que en los de tercer año fue de tres.

Haber realizado una rotación fuera del país estuvo asociado a una mayor frecuencia de publicación de algún artículo científico, lo cual se explica por el estímulo académico que encuentra el residente fuera del país, donde es probable que estos residentes tengan contacto con docentes investigadores con una alta producción científica [25].

La asociación de una mayor producción científica y mayor satisfacción global con su residencia coincide con una investigación que mostró que la implementación de un programa de investigación entre residentes de medicina interna aumentó la producción científica de ese servicio y tuvo impacto en la satisfacción del residente respecto a su sede docente [8]. De igual forma, entre residentes de segundo año de un hospital en Japón, las actividades de investigación estuvieron asociadas a una mayor satisfacción general en su programa de residencia [4]. Asimismo, entre residentes peruanos de Lima y Callao, se encontró que el apoyo de las universidades para realizar trabajos de investigación estuvo asociado con una mejor calificación de su residencia [2]. Esto sugiere que la formación en investiga-
Tabla II. Descripción de la muestra estudiada $(n=1.062)$.

\begin{tabular}{|c|c|c|c|}
\hline \multirow{9}{*}{$\begin{array}{l}\text { Variables } \\
\text { sociodemográficas }\end{array}$} & \multirow{2}{*}{ Sexo } & Masculino & $614(57,8 \%)$ \\
\hline & & Femenino & $448(42,2 \%)$ \\
\hline & \multirow{3}{*}{ Edad } & 24-29 años & $370(34,8 \%)$ \\
\hline & & 30-34 años & $393(37,0 \%)$ \\
\hline & & 35-55 años & $299(28,2 \%)$ \\
\hline & \multirow{2}{*}{$\begin{array}{l}\text { Soporta dependientes } \\
\text { económicos }\end{array}$} & No & $300(28,3 \%)$ \\
\hline & & Sí & $762(71,8 \%)$ \\
\hline & \multirow{2}{*}{ Tiene especialidad previa } & No & $1.009(95,4 \%)$ \\
\hline & & Sí & $49(4,6 \%)$ \\
\hline \multirow{7}{*}{$\begin{array}{l}\text { Variables de } \\
\text { universidad y } \\
\text { sede docente }\end{array}$} & \multirow{4}{*}{ Tipo de universidad } & Lima (pública) & $307(29,0 \%)$ \\
\hline & & Lima (privada) & $370(35,0 \%)$ \\
\hline & & Provincia (pública) & $237(22,4 \%)$ \\
\hline & & Provincia (privada) & $144(13,6 \%)$ \\
\hline & \multirow{3}{*}{ Institución de la sede } & MINSA y gobiernos regionales & $697(65,9 \%)$ \\
\hline & & EsSalud (seguro social) & $284(26,8 \%)$ \\
\hline & & Otros & $77(7,3 \%)$ \\
\hline \multirow{13}{*}{$\begin{array}{l}\text { Variables de } \\
\text { la residencia }\end{array}$} & \multirow{2}{*}{ Modalidad } & Libre & $865(81,8 \%)$ \\
\hline & & Destaque o cautiva & $193(18,2 \%)$ \\
\hline & \multirow{3}{*}{ Tipo de especialidad } & Clínica & $523(49,4 \%)$ \\
\hline & & Quirúrgica & $374(35,4 \%)$ \\
\hline & & Otras & $161(15,2 \%)$ \\
\hline & \multirow{3}{*}{ Año en curso } & Primero & $433(40,9 \%)$ \\
\hline & & Segundo & $287(27,1 \%)$ \\
\hline & & Tercero o más & $338(32,0 \%)$ \\
\hline & \multirow{3}{*}{$\begin{array}{l}\text { Horas que trabaja al } \\
\text { día sin contar guardias }\end{array}$} & $\leq 8 \mathrm{~h}$ & $312(31,3 \%)$ \\
\hline & & $>8 a<13 h$ & $598(60,0 \%)$ \\
\hline & & $\geq 13 h$ & $86(8,6 \%)$ \\
\hline & \multirow{2}{*}{$\begin{array}{l}\text { Ha realizado alguna } \\
\text { rotación fuera del país }\end{array}$} & No & $945(89,0 \%)$ \\
\hline & & Sí & $117(11,0 \%)$ \\
\hline \multirow{3}{*}{$\begin{array}{l}\text { Percepción } \\
\text { del residente }\end{array}$} & \multirow{3}{*}{$\begin{array}{l}\text { Opinión global del } \\
\text { proceso de formación }\end{array}$} & 0-5 puntos & $450(45,8 \%)$ \\
\hline & & 6-7 puntos & $435(44,3 \%)$ \\
\hline & & 8-9 puntos & $97(9,9 \%)$ \\
\hline \multirow{2}{*}{$\begin{array}{l}\text { Síntomas } \\
\text { depresivos }\end{array}$} & \multirow{2}{*}{$\begin{array}{l}\text { Patient Health } \\
\text { Questionnaire-2 }^{\text {a }}\end{array}$} & No & $584(59,2 \%)$ \\
\hline & & Sí & $403(40,8 \%)$ \\
\hline
\end{tabular}

MINSA: Ministerio de Salud. a Usando un punto de corte $\geq 2$. 
Tabla III. Factores asociados a haber publicado algún artículo durante la residencia.

\begin{tabular}{|c|c|c|c|c|c|c|}
\hline & & & \multicolumn{2}{|c|}{$\begin{array}{l}\text { Artículos publicados } \\
\text { durante la residencia }\end{array}$} & \multirow{2}{*}{$\begin{array}{l}\text { RP cruda } \\
\text { (IC 95\%) }\end{array}$} & \multirow{2}{*}{$\begin{array}{l}\text { RP ajustada } \\
\text { (IC 95\%) }\end{array}$} \\
\hline & & & 0 & $\geq 1$ & & \\
\hline \multirow{9}{*}{$\begin{array}{l}\text { Variables } \\
\text { sociodemográficas }\end{array}$} & \multirow{2}{*}{ Sexo } & Masculino & $536(87,3 \%)$ & $78(12,7 \%)$ & & \\
\hline & & Femenino & $408(91,1 \%)$ & $40(8,9 \%)$ & $0,70(0,49-1,01)$ & $0,69(0,48-1,00)$ \\
\hline & \multirow{3}{*}{ Edad } & 24-29 años & $329(88,9 \%)$ & $41(11,1 \%)$ & & \\
\hline & & 30-34 años & $334(85,0 \%)$ & $59(15,0 \%)$ & $1,35(0,93-1,97)$ & $1,06(0,71-1,58)$ \\
\hline & & 35-55 años & $281(94,0 \%)$ & $18(6,0 \%)$ & $0,54(0,32-0,93)$ & $0,42(0,24-0,73)$ \\
\hline & \multirow{2}{*}{$\begin{array}{l}\text { Soporta dependientes } \\
\text { económicos }\end{array}$} & No & $259(86,3 \%)$ & $41(13,7 \%)$ & & \\
\hline & & Sí & $685(89,9 \%)$ & $77(10,1 \%)$ & $0,74(0,52-1,05)$ & \\
\hline & \multirow{2}{*}{$\begin{array}{l}\text { Tiene especialidad } \\
\text { previa }\end{array}$} & No & $898(89,0 \%)$ & $111(11,0 \%)$ & & \\
\hline & & Sí & $43(87,8 \%)$ & $6(12,2 \%)$ & $1,11(0,52-2,40)$ & \\
\hline \multirow{7}{*}{$\begin{array}{l}\text { Variables de } \\
\text { universidad y } \\
\text { sede docente }\end{array}$} & \multirow{4}{*}{ Tipo de universidad } & Lima (pública) & $268(87,3 \%)$ & $39(12,7 \%)$ & & \\
\hline & & Lima (privada) & $327(88,4 \%)$ & $43(11,6 \%)$ & $0,91(0,61-1,37)$ & \\
\hline & & Provincia (pública) & $217(91,6 \%)$ & $20(8,4 \%)$ & $0,66(0,40-1,11)$ & \\
\hline & & Provincia (privada) & $129(89,6 \%)$ & $15(10,4 \%)$ & $0,82(0,47-1,44)$ & \\
\hline & \multirow{3}{*}{ Institución de la sede } & MINSA y gobiernos regionales & $626(89,8 \%)$ & $71(10,2 \%)$ & & \\
\hline & & EsSALUD (seguro social) & $248(87,3 \%)$ & $36(12,7 \%)$ & $1,24(0,85-1,81)$ & \\
\hline & & Otros & $67(87,0 \%)$ & $10(13,0 \%)$ & $1,27(0,69-2,37)$ & \\
\hline \multirow{13}{*}{$\begin{array}{l}\text { Variables de } \\
\text { la residencia }\end{array}$} & \multirow{2}{*}{ Modalidad } & Libre & $763(88,2 \%)$ & $102(11,8 \%)$ & & \\
\hline & & Destaque o cautiva & $178(92,2 \%)$ & $15(7,8 \%)$ & $0,66(0,39-1,11)$ & \\
\hline & \multirow{3}{*}{$\begin{array}{l}\text { Tipo de } \\
\text { especialidad }\end{array}$} & Clínica & $453(86,6 \%)$ & $70(13,4 \%)$ & & \\
\hline & & Quirúrgica & $341(91,2 \%)$ & $33(8,8 \%)$ & $0,66(0,45-0,98)$ & $0,67(0,44-1,01)$ \\
\hline & & Otras & $147(91,3 \%)$ & $14(8,7 \%)$ & $0,65(0,38-1,12)$ & $0,69(0,41-1,18)$ \\
\hline & \multirow{3}{*}{ Año en curso } & Primero & $405(93,5 \%)$ & $28(6,5 \%)$ & & \\
\hline & & Segundo & $257(89,5 \%)$ & $30(10,5 \%)$ & $1,62(0,99-2,65)$ & $1,68(1,03-2,72)$ \\
\hline & & Tercero a más & $279(82,5 \%)$ & $59(17,5 \%)$ & $2,70(1,76-4,14)$ & $1,98(1,20-3,26)$ \\
\hline & \multirow{3}{*}{$\begin{array}{l}\text { Horas que trabaja al } \\
\text { día sin contar guardias }\end{array}$} & $\leq 8 \mathrm{~h}$ & $277(88,8 \%)$ & $35(11,2 \%)$ & & \\
\hline & & $>8 \mathrm{a}<13 \mathrm{~h}$ & $534(89,3 \%)$ & $64(10,7 \%)$ & $0,95(0,65-1,41)$ & \\
\hline & & $\geq 13 \mathrm{~h}$ & 75 (87,2\%) & $11(12,8 \%)$ & $1,14(0,60-2,15)$ & \\
\hline & \multirow{2}{*}{$\begin{array}{l}\text { Ha realizado alguna } \\
\text { rotación fuera del país }\end{array}$} & No & $853(90,3 \%)$ & $92(9,7 \%)$ & & \\
\hline & & Sí & $91(77,8 \%)$ & $26(22,2 \%)$ & $2,28(1,54-3,37)$ & $1,62(1,02-2,58)$ \\
\hline \multirow{3}{*}{$\begin{array}{l}\text { Percepción } \\
\text { del residente }\end{array}$} & \multirow{3}{*}{$\begin{array}{l}\text { Opinión global del } \\
\text { proceso de formación }\end{array}$} & 0-5 puntos & $417(92,7 \%)$ & $33(7,3 \%)$ & & \\
\hline & & 6-7 puntos & $377(86,7 \%)$ & $58(13,3 \%)$ & $1,82(1,21-2,73)$ & $1,57(1,04-2,38)$ \\
\hline & & 8-9 puntos & $79(81,4 \%)$ & $18(18,6 \%)$ & $2,53(1,49-4,30)$ & $1,98(1,13-3,48)$ \\
\hline \multirow{2}{*}{$\begin{array}{l}\text { Síntomas } \\
\text { depresivos }\end{array}$} & \multirow{2}{*}{$\begin{array}{l}\text { Patient Health } \\
\text { Questionnaire-2 }\end{array}$} & No & $504(86,3 \%)$ & $80(13,7 \%)$ & & \\
\hline & & Sí & $374(92,8 \%)$ & $29(7,2 \%)$ & $0,53(0,35-0,79)$ & $0,63(0,41-0,96)$ \\
\hline
\end{tabular}

IC 95\%: intervalo de confianza al 95\%; MINSA: Ministerio de Salud; RP: razón de prevalencia. 
ción y la posibilidad de publicar artículos científicos durante la residencia pueden ser un punto importante para la satisfacción de los residentes con su formación.

La mayor edad del residente estuvo asociada a una menor prevalencia de haber publicado algún artículo, probablemente debido a que este grupo ha culminado el pregrado varios años atrás, por lo que podría ser que les resulte más difícil adaptarse a las actividades asistenciales de la residencia, dificultad que se traduciría en menos tiempo para desarrollar actividades extracurriculares como las relacionadas con la investigación. De igual forma, el hecho de presentar síntomas depresivos puede condicionar una menor disposición a las actividades de la residencia, incluyendo las de investigación [26].

El sexo femenino estuvo asociado a una menor producción científica. Esta disparidad de producción científica por sexo se ha descrito también en el mundo [27]. Por cada mujer que figura como primera autora en una publicación científica, existen cerca de dos hombres. Esta disparidad no es homogénea y se encuentra que en algunas regiones, como América del Sur y Europa del este, hay más paridad de género, o incluso existen países, como Macedonia o Sri Lanka, entre otros, donde las mujeres lideran la producción científica. No existen razones probadas para esta disparidad, y los estudios que la han sugerido tienen sesgos de evaluación, como el tiempo destinado a la maternidad y la crianza. Sin embargo, es posible que las mujeres tiendan a ser subestimadas por los equipos de investigación, entre otras dificultades para llegar a una alta producción científica [27].

Entre otros factores encontrados asociados a una menor producción científica entre los residentes en otros estudios destacan el tiempo disponible para la investigación, oportunidades e interés personal en la investigación, fondos para investigación y un programa de apoyo para la residencia [15,28]. Al menos entre nuestros residentes, el número de horas trabajadas no se asoció a la producción científica, lo que sugiere que el tiempo disponible podría no ser una limitante para las actividades de investigación; no obstante, no hemos evaluado cuántas de las horas laborales se dedican a investigación, por lo cual estos resultados deben tomarse con cautela.

\section{Limitaciones}

Dentro de las limitaciones de nuestro estudio destaca que nuestra población participó de forma voluntaria en la encuesta, lo cual puede hacer que el número de encuestados no represente el universo de residentes en Perú o de cada región, universidad o sede, y por lo cual los hallazgos no puedan generalizarse. Asimismo, la información otorgada, al ser mediante una encuesta virtual, no puede verificarse, sobre todo en la variable desenlace de producción científica de los residentes, por lo que ésta puede haberse sobreestimado. Finalmente, es posible que no se hayan incluido otras variables que puedan potencialmente influenciar en las actividades de investigación y publicación de los residentes, como es el caso del apoyo económico para ejecutar proyectos de investigación y su posterior publicación.

Sin embargo, y a pesar de las limitaciones observadas, éste es el primer estudio peruano sobre publicación y producción científica de residentes realizado a nivel nacional que nos permite tener una aproximación de los aspectos estudiados.

En conclusión, aproximadamente uno de cada 10 residentes manifestó haber publicado algún artículo científico durante la residencia. Esto fue menos prevalente en las mujeres, en los residentes de mayor edad, en quienes cursaban años inferiores de la residencia, en quienes no realizaron ninguna rotación fuera del país, en quienes tuvieron una peor opinión del proceso de formación y en quienes presentan síntomas depresivos.

\section{Bibliografía}

1. Herrera-Añazco P, Hernández AV, Sánchez-Rivas F, AranaMaestre C. Percepción de la formación durante el residentado médico en hospitales del Ministerio de Salud de Lima, Perú. Revista Peruana de Medicina Experimental y Salud Pública 2014; 31: 606-7.

2. Miní E, Medina J, Peralta V, Rojas L, Butrón J, Gutiérrez EL. Programa de residentado médico: percepciones de los médicos residentes en hospitales de Lima y Callao. Revista Peruana de Medicina Experimental y Salud Pública 2015; 32: 303-10.

3. Herrera-Añazco P, Bonilla-Vargas L, Hernández AV, Silveira-Chau M. Perception of physicians about medical education received during their Nephrology residency training in Peru. Jornal Brasileiro de Nefrologia 2015; 37: 333-40.

4. Takahashi O, Ohde S, Jacobs JL, Tokuda Y, Omata F, Fukui T. Residents' experience of scholarly activities is associated with higher satisfaction with residency training. J Gen Intern Med 2009; 24: 716-20.

5. Valle R, Perales A. Nueva normativa de titulación en el residentado médico en el Perú: problemas y perspectivas. Revista Peruana de Medicina Experimental y Salud Pública 2016; 33: 357-61.

6. Seaburg LA, Wang AT, West CP, Reed DA, Halvorsen AJ, Engstler G, et al. Associations between resident physicians' publications and clinical performance during residency training. BMC Med Educ 2016; 16: 22.

7. Sansone RA, Wiederman MW, Sawyer RJ. Effective research strategies for trainees in internal medicine residency programs. Prim Care Companion CNS Disord 2015; 17. doi: 10.4088/ PCC.14r01712.

8. Rothberg MB, Kleppel R, Friderici JL, Hinchey K. Implementing a resident research program to overcome barriers to resident research. Acad Med 2014; 89: 1133-9.

9. Gaught AM, Cleveland CA, Hill JJ 3rd. Publish or perish?: 
physician research productivity during residency training. Am J Phys Med Rehabil 2013; 92: 710-4.

10. Ullrich N, Botelho CA, Hibberd P, Bernstein HH. Research during pediatric residency: predictors and resident-determined influences. Acad Med 2003; 78: 1253-8.

11. West CP, Halvorsen AJ, McDonald FS. Scholarship during residency training: a controlled comparison study. Am J Med 2011; 124: 983-7. e1.

12. Kanna B, Deng C, Erickson SN, Valerio JA, Dimitrov V, Soni A. The research rotation: competency-based structured and novel approach to research training of internal medicine residents. BMC Med Educ 2006; 6: 52.

13. Vázquez II, Bran DR, Fernández MM, Valcárcel PV, Rey RR, Monte R. Actividad investigadora de los residentes de medicina interna de Galicia. Galicia Clínica 2012; 73: 7-10.

14. Manjarin M, Cutri AM, Torres FA, Noguerol ME, Ossorio MF, Duran P, et al. Evaluación de la producción científica en la residencia de pediatría. Arch Argent Pediatr 2009; 107: 26-9.

15. Ticse R, Ygreda P, Samalvides F. Publicación de los proyectos de investigación para la titulación como médicos especialistas de una universidad peruana, 2007-2010. Revista Peruana de Medicina Experimental y Salud Pública 2014; 31: 292-6.

16. Herrera-Añazco P, Mezones-Holguín E, Hernández AV. La investigación científica es deficiente entre residentes de nefrología: el caso peruano. Revista de Nefrología, Diálisis y Trasplante 2014; 34: 159-61.

17. Valle R, Perales A. Investigación en salud en el Perú: mucho por hacer y cambiar. Revista Peruana de Medicina Experimental y Salud Pública 2016; 33: 833-4.

18. Hernández-Asensio R. ¿Quién escribe más y sobre qué? Cambios recientes en la geopolítica de la producción científica en América Latina y el Caribe. Argumentos 2014; 4: 51-63.

19. Rodríguez JA, Galán-Rodas E, Núñez M, Maguiña C. Acciones en el fomento de la investigación médica en el Perú. Revista Peruana de Medicina Experimental y Salud Pública 2010; 27: 654 .
20. Arroll B, Goodyear-Smith F, Crengle S, Gunn J, Kerse N, Fishman T, et al. Validation of PHQ-2 and PHQ-9 to screen for major depression in the primary care population. Ann Fam Med 2010; 8: 348-53.

21. Mitwalli H, Al Ghamdi K, Moussa N. Perceptions, attitudes, and practices towards research among resident physicians in training in Saudi Arabia. East Mediterr Health J 2014; 20: 99.

22. Pereyra-Elías R, Huaccho-Rojas JJ, Taype-Rondan A, Mejía CR Mayta-Tristán P. Publicación y factores asociados en docentes universitarios de investigación científica de escuelas de medicina del Perú. Revista Peruana de Medicina Experimental y Salud Pública 2014; 31: 424-30.

23. Manring MM, Panzo JA, Mayerson JL. A framework for improving resident research participation and scholarly output. J Surg Educ 2014; 71: 8-13.

24. Ticse R, Pamo O, Samalvides F, Quispe T. Factores asociados a la culminación del proyecto de investigación requerido para optar el título de especialista en una universidad peruana. Revista Peruana de Medicina Experimental y Salud Pública 2014; 31: 48-55.

25. Polasek O, Kolcic I, Buneta Z, Cikes N, Pecina M. Scientific production of research fellows at the Zagreb University School of Medicine, Croatia. Croat Med J 2006; 47: 776-82.

26. Bernburg M, Vitzthum K, Groneberg DA, Mache S. Physicians occupational stress, depressive symptoms and work ability in relation to their working environment: a cross-sectional study of differences among medical residents with various specialties working in German hospitals. BMJ Open 2016; 6: e011369.

27. Sugimoto CR, Lariviere V, Ni C, Gingras Y, Cronin B. Global gender disparities in science. Nature 2013; 504: 211-3.

28. Levine RB, Hebert RS, Wright SM. Resident research and scholarly activity in internal medicine residency training programs. J Gen Intern Med 2005; 20: 155-9. 